\title{
Growth Rates of Vaccinated and Unvaccinated Weaner Angus Cattle in BVDV Endemic Herds in Southern NSW
}

\author{
Allworth $\mathrm{MB}^{1,2,3 *}$, Smith $\mathrm{AK}^{1}$ and Prescott $\mathrm{M}^{4}$ \\ ${ }^{1}$ School of Animal and Veterinary Sciences, Charles Sturt University, Australia \\ ${ }^{2}$ Fred Morley Centre, School of Animal and Veterinary Sciences, Australia \\ ${ }^{3}$ Graham Centre for Agricultural Innovation (NSW Department of Primary Industries and \\ Charles Sturt University), Australia \\ ${ }^{4}$ Quantitative Consulting Unit, Charles Sturt University, Wagga Wagga, NSW, Australia
}

Research Article

Volume 5 Issue 1

Received Date: July 14, 2020

Published Date: August 10, 2020

DOI: $10.23880 /$ oajvsr-16000195

*Corresponding author: Allworth MB, School of Animal and Veterinary Sciences, Charles Sturt University, Locked Bag 588, Wagga Wagga, NSW 2678, Australia, Email: ballworth@csu.edu.au

\section{Abstract}

In Australia Bovine Viral Diarrhoea Virus (BVDV) is considered to be primarily associated with reproductive losses in cattle. Trials in two BVDV endemically infected beef herds in southern NSW were undertaken to assess the impact of exposure to BVDV on weight gain in young recently weaned cattle. The study, comprising 410, 6-15 month old animals (Property 1), and 120, 7-15 month old animals (Property 2), compared growth rates between vaccinated and control animals during ongoing exposure to known persistently-infected individuals. At the commencement of the trial, 70\% (Property 1) and 68\% (Property 2) of the animals were antibody-positive. At the end of the trial, almost all animals were antibody-positive, either as a result of natural exposure or vaccination, or both. Predicted mean weight gain for Vaccinate animals compared to Control animals was $0.25 \mathrm{~kg}$ lower on Property 1 and $4.2 \mathrm{~kg}$ greater on Property 2, these differences being non-significant. Comparison of animals which consistently tested antibody positive and those animals that seroconverted from antibody negative to antibody positive did not show any significant difference in weight gains. The results from this trial provide evidence that vaccination against BVDV in young (6-15 months) beef cattle in endemic herds grazing at pasture does not result in increased growth rates.

Keywords: BVDV; Pestivirus; Beef cattle; Weight gain; Vaccination

\section{Introduction}

The Bovine Viral Diarrhoea Virus (BVDV) is an RNA virus of the Pestivirus species, within the family Flaviviridae, which has worldwide distribution and causes a range of reproductive disorders and immunosuppressive effects [1]. A persistently-infected (PI) calf, resulting from infection with the virus during early pregnancy (days 18-120) in a BVDVimmunologically-naïve dam, when the developing foetus is still not immunocompetent, can subsequently shed large volumes of virus, from all body secretions, for the remainder of its life [2]. These PI calves, generally, only occur at a low prevalence $(0.5-2 \%)$, but are considered to be the major source of infection for other animals [3-5].

BVDV has been estimated to cost the Australian cattle industry $\$ 114.4 \mathrm{M}$ p.a., placing it amongst the top two economically important cattle diseases in Australia [6]. It has been suggested that young grazing cattle exposed to BVDV may suffer weight losses (Holmes and McGowan, unpublished), and evidence in feedlots suggest that weight gains may be decreased by exposure to BVDV, although these losses are generally considered to be due to respiratory diseases resulting from immunosuppression caused by 


\section{Open Access Journal of Veterinary Science \& Research}

BVDV [7,8]. Vaccination of animals prior to joining has been recommended to control pestivirus and minimise its reproductive consequences within a herd. An alternate strategy for control has been to deliberately expose young heifers to PI animals several months before joining. There have been no published reports of either the effect of exposure to BVDV on weight gains in young cattle grazing in endemic herds, or whether vaccination mitigates against any such effects.

The purpose of this investigation was to examine the impact of natural viral challenge, for both vaccinated and non-vaccinated animals, on growth rates of weaner cattle in two endemic herds.

\section{Materials and Methods}

Two properties in southern NSW were selected, based on their contact with CSU's School of Animal and Veterinary Sciences. Property 1 was located east of Holbrook, and BVDV was diagnosed on the property in September 2012. No control measures had been instituted. Property 2 was located east of Wagga Wagga, and BVDV had been present in the herd for a number of years. Control on this second property involved the deliberate exposure of heifers each year with a PI for 2-3 months post-weaning.

Both herds are winter/spring calving Angus herds. Animals are grazed at pasture all year round at typical local stocking rates eg (12-18 DSE/ha). These trials were approved by the CSU Animal Ethics Committee (No 15/012; No 15/014).

\section{Property 1}

Prior to the commencement of the trial (10 Feb 2015, Day -29), four hundred and ten, 5 to 6 month old Angus calves, still suckling on their dams, were weighed and blood sampled to determine BVDV antibody status ('Initial' sample). The animals were allocated to one of two groups (Control or Vaccinate), stratified by sex (heifer or steer), antibody status (negative or positive), dam age, and bodyweight. At the commencement of the trial (11 Mar 2015, Day 0) and 4 weeks later (8 Apr 2015, Day 28), all calves were weighed and those in the Vaccinate group received $2 \mathrm{mls}$ of BVDV vaccine subcutaneously (Pestigard $\AA$, Zoetis, Australia), as per manufacturers recommendations.

Calves were yard-weaned on 22 Apr 2015 (Day 42), with ad lib access to lucerne hay. All calves received 2 mls 5-in- 1 clostridial vaccine (Ultravac $®, Z o e t i s, ~ A u s t r a l i a)$ (their second dose) and 30mls doramectin anthelmintic (Dectomax® Cattle Pour-On, Zoetis, Australia) on 27 Apr 2015 (Day 47).
The calves remained in the weaning yards until 1 May 2015 (Day 51), when they were drafted into two mobs (steers and heifers). Each mob was then managed in separate paddocks for the duration of the trial, with the heifer calves being grazed in one of three paddocks, and the steer calves one of four paddocks. Animals in both mobs were weighed on 15 June 2015 (Day 96), 16 September 2015 (Day 189) and 23 November 2015 (Day 257). In addition, animals were blood sampled as follows:

- $\quad$ on $15^{\text {th }}$ June 2015 (Days 96, 'Interim' sample), all animals

- on 16 September 2015 (Day 189), a sample of animals (animals that had a S/P ratio (see below) at or below 0.65 at the June sampling and $10 \%$ of animals with S/P ratios above 0.65 at the June sampling)

- $\quad$ on $23^{\text {rd }}$ November 2015 (Day 257,'Final' sample), all animals.

Animals were fed lucerne hay once/week from late May until early August to help animals maintain weight. All animals received 30mls doramectin anthelmintic (Dectomax® Cattle Pour-On, Zoetis, Australia) on 29 July 2015 (Day 140).

\section{Property 2}

One hundred and twenty 7-8 month old weaned Angus heifers were systematically allocated to one of two groups (Control or Vaccinate). Heifers were weighed and blood sampled on 19 March 2015 (Day 0, 'Initial' sample) and at the end of the trial on 30 September 2015 (Day 195, 'Final' sample). Heifers in the Vaccinate group received $2 \mathrm{mls}$ of BVDV vaccine subcutaneously (Pestigard®, Zoetis, Australia), as per manufacturers recommendations on both Day 0 and Day 28 (16 April 2015).

\section{Both Properties}

Plain blood samples were collected, by tail-vein venepuncture, in $4 \mathrm{~mL}$ Vacuettes ${ }^{\circledR}$ (Greiner Bio-one $\mathrm{GmbH}$, Austria), stored on ice at the time of collection, refrigerated overnight at $4^{\circ} \mathrm{C}$, then shipped on ice to Swan Vet Services' laboratory, Esperance, WA. Shipping was usually completed within 48 hours of despatch. Sera was collected from the samples and assayed for serum BVDV antibody levels by ELISA (IDEXX BVDV Total Ab Test, IDEXX Laboratories and Australia) as per the manufacturer's specified protocol.

Results for the ELISA BVDV Total Ab test were recorded as sample-to-positive $(\mathrm{S} / \mathrm{P})$ ratios. For the purposes of this trial, animals were considered to be seronegative if values were recorded as an S/P ratio less than 0.3 , and seropositive if the S/P ratio was equal to, or greater than, 0.3. The ELISA sensitivity and specificity for this assay, conducted at Swan Vet Services' laboratory, have been previously reported as 


\section{Open Access Journal of Veterinary Science \& Research}

$96.7 \%$ and $97.1 \%$, respectively $[9,10]$.

Blood samples that were recorded with a S/P ratio less than 0.65 at the June, September and November samplings for Property 1 and both samplings for Property 2 were also tested for BVDV antigen by BVDV antigen-capture ELISA (IDEXX BVDV Ag/Serum Plus, IDEXX Laboratories, Australia) as per manufacturer's instructions. Results for the ELISA BVDV Ag/Serum Plus were reported as Optical Density (OD) values, with a positive result being any value greater than 0.7 and considered to be diagnostic for a PI animal. The sensitivity and specificity of this test are each $100 \%$ as per the manufacturer's validation report.

Antibody status was reported based on whether antibody results remained the same or changed between samples, with the following statuses being assigned: stable positive for animals that tested antibody positive throughout the trial, stable negative for animals that tested antibody negative during the trial (and were also antigen negative (antigen positive PI animals were removed from the analysis)), seroconverters for animals that changed from antibody negative to antibody positive, and waning for animals that at any point changed from antibody positive to antibody negative.
Statistical analysis for weight gains was performed using Stata 12 [11]. Weight gain was analysed using a linear mixed model, with weight gain being the response variable and treatment (Vaccinate compared to Control) being the main explanatory variable. For Property 1 , sex, time and antibody status were covariates, with paddock, dam age and mob being random effects. Statistical significance for $\mathrm{p}<0.05$ was determined using Bonferroni correction to account for multiple pairwise comparisons made. For Property 2, antibody status was a covariate. Weight gains are reported as predicted means from the model.

Differences in deaths were analysed using the z-test for proportions.

\section{Results}

Of the 410 animals sampled at the commencement of the trial on Property 1 and the 120 animals on Property 2, $70 \%$ of trial animals on Property 1 and $69 \%$ of trial animals on Property 2 were antibody positive (S/P ratio $\geq 0.3$ ) (Table 1).Two PI animals on Property 1 ( $0.5 \%$ of animals), and one on Property 2 ( $0.8 \%$ of animals) were identified, confirming that both herds were endemically infected with BVDV.

\begin{tabular}{|c|c|c|c|}
\hline \multicolumn{2}{|c|}{ Number in Mob } & Total 'Positive’ for BVDV antibody (\%) & Total 'Negative' for BVDV antibody (\%) \\
\hline \multicolumn{4}{|c|}{ Property 1} \\
\hline Total & 410 & $285(70 \%)$ & $125(30 \%)$ \\
\hline \multicolumn{4}{|c|}{ Property 2} \\
\hline Total & 120 & $83(69 \%)$ & $37(31 \%)$ \\
\hline
\end{tabular}

Table 1: Initial distribution of BVDV antibody serology results on each property.

From the two groups of animals that commenced the trial on both properties (Property 1, 410; Property 2,120) there were 395 and 118 complete sets of records, respectively, available for analysis.

For Properties 1 and 2, almost all animals ( 275 out of 278 and 82 out of 83 respectively) that were antibody positive at the commencement of the trial remained antibody positive (stable positive) and similarly almost all animals (116 out of 117 and 27 out of 35 respectively) that were antibody negative at the commencement of the trial were antibody positive at the end (seroconverted).The distribution of the assigned antibody statuses is given in Table 2 .

\begin{tabular}{|c|c|c|c|c|c|c|}
\hline & \multicolumn{3}{|c|}{ Property 1 } & \multicolumn{3}{c|}{ Property 2 } \\
\cline { 2 - 7 } & Control & Vaccine & Total & Control & Vaccine & Total \\
\hline Stable negative & 1 & 1 & 2 & 4 & 4 & 8 \\
\hline Stable positive & 135 & 140 & 275 & 39 & 43 & 82 \\
\hline Seroconverted & 59 & 58 & 117 & 16 & 11 & 27 \\
\hline Waning & 1 & 0 & 1 & 1 & 0 & 1 \\
\hline & 196 & 199 & 395 & 60 & 58 & 118 \\
\hline
\end{tabular}

Table 2: Assigned antibody status. 


\section{Open Access Journal of Veterinary Science \& Research}

At the commencement of the trial, animals averaged $231 \mathrm{~kg}$ on Property 1 and $239 \mathrm{~kg}$ on Property 2, while at the conclusion of the trial, animals had gained $126 \mathrm{~kg}$ to average $357 \mathrm{~kg}$ on Property 1 and $101 \mathrm{~kg}$ to average $340 \mathrm{~kg}$ on Property 2.

For Property 1, steers gained significantly more weight $(6.8 \mathrm{~kg})$ than heifers as expected, but there was no interaction between treatment and sex, so combined results for heifers and steers are reported. For both properties, there were small non-significant differences in weight gains between Vaccinate and Control animals. Predicted mean weight gain for Vaccinate animals compared to Control animals was 0.25 kg lower on Property 1 and $4.2 \mathrm{~kg}$ greater on Property 2 (Table 3). For Property 1, there was no interaction between time and treatment, so differences in predicted mean weight gains were similar for each time period analyzed (Table 3).

\begin{tabular}{|c|c|c|c|c|}
\hline Property & Time period & Vaccinate & Control & [Vaccinate -Control]* \\
\hline 1 & Mar-Jun & -37.4 & -37.1 & \\
\hline 1 & Jun-Sep & 40.7 & 120.8 & -0.25 \\
\hline 1 & Sep-Nov & 120.6 & 124.5 & 4.2 \\
\hline
\end{tabular}

Table 3: Predicted mean weight changes by Treatment group (Vaccinate or Control) and difference (Vaccinate-Control) for both Properties and for specific time periods for Property 1.

* Differences not significant.

For Property 1, antibody status had a significant effect on weight gain, and there was a significant interaction between treatment and antibody status, with seronegative control animals having significantly greater weight gains. However, the very low numbers of seronegative animals meant that this result is not considered valid. Pairwise comparisons of Control animals for the two antibody status groups with sufficient animals for a reasonable comparison (stable positive and seroconverted) indicated that despite animals which seroconverted having greater weight gain than seropositive animals ( $5.1 \mathrm{~kg}$ overall), the difference was not significant (Table 4).

\begin{tabular}{|c|c|c|c|c|}
\hline Property & Time period & Stable Positive & Seroconvert & [STP-SC $^{*}$ \\
\hline 1 & Mar-Jun & -38 & -35.2 & -2.8 \\
\hline 1 & Jun-Sep & 39 & 45.6 & -6.6 \\
\hline 1 & Sep-Nov & 121.2 & 119.5 & 1.7 \\
\hline 2 & Mar- Nov & 122.8 & 127.9 & -5.1 \\
\hline
\end{tabular}

Table 4: Predicted mean weight changes for Control animals by Antibody status (Stable positive (STP) or Seroconverted (SC)) and difference (STP-SC) for both Properties and for specific time periods for Property 1.

* Differences not significant.

From the 410 animals that were initially weighed and blood sampled on Property 1, in February 2015, 399 animals were present on 23 November 2015. One animal died between initial weighing and vaccination. This animal was antibody negative but was not antigen tested. Excluding this animal, for the ten animals that died during the trial, seven were Control animals and three were Vaccinate, with this difference being non-significant $(\mathrm{p}=0.28)$. Five animals died from bloat, one of the two known PI animals died, and the cause of death of the other four animals was not identified, although given their antibody results it was unlikely to be directly associated with BVDV (all four animals were antibody positive on two successive samplings prior to death). On Property 2, two animals died during the trial. Both were in the Vaccinate group, one was the PI animal (which died in the last week of the trial), and the other was an animal that was BVDV antibody-positive at the commencement of the trial.

\section{Discussion}

The results from this trial provide evidence that vaccination against BVDV in young (6 - 15 months) beef 


\section{Open Access Journal of Veterinary Science \& Research}

cattle in endemic herds grazing at pasture does not result in increased growth rates.

The aim of this trial was to assess the impact of exposure to pestivirus challenge in young growing beef animals grazing at pasture. To this end, half the animals were vaccinated under the assumption that this vaccination may be protective against any effects of viral challenge on growth rate. Further, it was expected that a reasonable proportion of unvaccinated animals would remain seronegative during the trial, and that comparison in weight gains between seronegative animals and those animals that seroconverted would provide an assessment of the effect of viral challenge on weight gain, and the degree to which vaccination provided protection against any such negative effect.

However, in both the herds monitored, the initial high rate $(70 \%)$ of seropositive animals, and the subsequent very high seroconversion rate of those animals which were initially seronegative, especially on Property 1 , reduced the valid comparisons which could be made. In an effort to more accurately assess the impact of exposure of BVDV on growth rates, comparisons were made between those animals that tested as seropositive on all test dates (stable positive) and those animals that had seroconverted from antibody negative to antibody positive (seroconverted). These comparisons indicated that there was no weight disadvantage in animals that had seroconverted; suggesting that exposure to BVDV did not impact on weight gains in these animals.

However, it was not possible to determine whether the initial seropositive animals were seropositive as a result of maternal antibodies, or as a result of earlier exposure to BVDV and active immunity. If the former was the case, then it is possible that animals that were initially seropositive may in fact have waned in antibody levels and then been exposed subsequently to BVDV and then seroconverted, yet being recorded as stable positive. If this occurred, then the comparisons using antibody status would not accurately represent the effects on weight gains. If, however, those animals that were antibody positive at the commencement of the trial had in fact already been exposed to BVDV and were immune, then it may be expected that continued exposure to BVDV would have less impact on these animals than those animals which were seronegative. If this was the case, the failure to detect any differences between stable positive and seroconverting animals would add weight to the findings that immunologically protected animals do not have any weight gain advantage over immunologically naïve animals.

While this study did not specifically measure the effect of BVDV exposure on weight gain, the findings that unvaccinated young recently-weaned Angus cattle had similar weight gains to vaccinated cattle on two different properties are consistent with the assumptions of Lane et al (2015) that BVDV does not result in any weight loss. These findings also are consistent with trials in Canadian feedlot animals which concluded exposure to PI animals did not affect weight gain $[12,13]$ although not consistent with other trials $[9,14]$.

In conclusion, the results from this trial suggest that vaccination in endemic herds, where one or more PIs are present among weaner cattle, does not result in any weightgain advantage in vaccinated animals.

\section{Acknowledgements}

The authors would like to thank the owners of the two properties for making the animals available for the trial, and for yarding the animals for measurements, Shawn McGrath for assistance with data collection, Zoetis Australia for providing the vaccine and financial support, IDEXX for supplying the ELISA reagents and blood collection equipment, Jess Turner (IDEXX) for logistical support, and Swans Veterinary Services for undertaking the assays for BVDV antibody and antigen.

\section{References}

1. Lanyon SR, Hill FI, Reichel MP, Joe Brownlie (2014) Bovine viral diarrhoea: Pathogenesis and diagnosis. The Veterinary Journal 199(2): 201-209.

2. Brownlie J (1990) Pathogenesis of mucosal disease and molecular aspects of bovine virus diarrhoea virus. Vet Microbiol 23(1-4): 371-382.

3. Houe H (1995) Epidemiology of bovine viral diarrhoea virus. Vet Clin Food Anim 11(3): 521-547.

4. Houe H (1999) Epidemiology features and economic importance of bovine viral diarrhoea virus BVDV) infections. Vet Microbiol 64(2-3): 89-107.

5. Lanyon SR, Reichel MP (2013) Understanding the impact and control of bovine viral diarrhoea in cattle populations. Springer Science reviews 1: 85-93.

6. Lane J, Jubb T, Shephard R, Webb-Ware, Jjohn, et al. (2015) Priority list of endemic diseases for the red meat industries. MLA Report B.AHE.0010, Meat \& Livestock Australia Ltd, Sydney, Australia.

7. Campbell JR (2004) Effect of bovine viral diarrhea virus in the feedlot. Vet Clin Food Anim 20(1): 39-50.

8. Hessman WE, Fulton RW, Sjeklocha DB, Timothy AM, Julia FR, et al. (2009) Evaluation of economic effects and the health and performance of the general cattle 


\section{Open Access Journal of Veterinary Science \& Research}

population after exposure to cattle persistently infected with bovine viral diarrhea virus in a starter feedlot. Am J Vet Res 70(1): 73-85.

9. Burciaga-Robles LO, Krehbiel CR, Step DL, Holland BP, Richards CJ, et al. (2010) Effects of exposure to calves persistently infected with bovine viral diarrhea virus type $1 \mathrm{~b}$ and Mannheima heaemolytica challenge on animal performance, nitrogen balance, and visceral organ mass in beef steers. J Anim Sci 88(6): 2179-2188.

10. Lanyon SR, Anderson ML, Bergman E, Reichel MP (2013) Validation and evaluation of a commercially available ELISA for the detection of antibodies specific to bovine viral diarrhoea virus (bovine pestivirus). Aust Vet J 91 (1-2): 52-56.

11. Stata Corp (2011) Stata Statistical Software: Release 12.
College Station, StataCorp LP, TX.

12. Booker CW, Abutarbush SM, Morley PS, Timothy Guichon P, Brian K Wildman, et al. (2008) The effect of bovine viral diarrhea virus infections on health and performance of feedlot cattle. Can Vet J 49(3): 253-260.

13. Elam NA, Thomson DU, Gleghorn JF (2008) Effects of long- or short-term exposure to a calf identified as persistently infected with bovine viral diarrhea virus on feedlot performance of freshly weaned, transportstressed beef heifers. J Anim Sci 86(8): 1917-1924.

14. Grooms DL, Brock KV, Bolin SR, Dale M Grotelueschen, Victor S Cortese (2014) Effect of constant exposure to cattle persistently infected with bovine viral diarrhea virus on morbidity and mortality rates and performance of feedlot cattle. JAVMA 244(2): 212-224. 